\title{
Stellarator Nonlinearly Saturated Periodicity-Breaking Ideal Magnetohydrodynamic Equilibrium States
}

\author{
W.A. Cooper ${ }^{1,2}$, D. López-Bruna ${ }^{3}$, M.A. Ochando ${ }^{3}$, F. Castejón ${ }^{3}$, J.P. Graves ${ }^{1}$, A. Kleiner ${ }^{1}$, \\ S. Lanthaler ${ }^{1}$, H. Patten ${ }^{1}$, M. Raghunathan ${ }^{1}$, J.M. Faustin ${ }^{4}$, and the TJ-II team ${ }^{3 *}$ \\ ${ }^{1}$ Ecole Polytechnique Fédérale de Lausanne (EPFL), \\ Swiss Plasma Center (SPC), Lausanne, CH-1015 Lausanne, Switzerland \\ ${ }^{2}$ Swiss Alps Fusion Energy (SAFE), CH-1864 Vers l'Eglise, Switzerland \\ ${ }^{3}$ Laboratorio Nacional de Fusión, CIEMAT, 28040 Madrid, Spain \\ ${ }^{4}$ Max Planck Institut für Plasmaphysik, Teilinstitut Greifswald, Greifswald, Germany
}

(Dated: August 22, 2018)

\begin{abstract}
The relaxation of the constraint of periodicity imposed by the external confining magnetic field coils in a nominally 4-field period Helias Advanced Stellarator configuration produces weak periodicity-breaking deformations of the plasma. The corrugations are driven by the interaction of the pressure gradient with the magnetic field line curvature and correspond to saturated ideal magnetohydrodynamic interchanges with a mode structure dominated by nonresonant $m=1, n= \pm 1$ Fourier components. The conditions of quasi-isodynamicity of the Helias reactor system investigated are not significantly altered by the periodicity-breaking distortions.
\end{abstract}

Magnetohydrodynamic (MHD) activity can alter the symmetry properties of magnetically confined plasma. A three-dimensional (3D) equilibrium solver [1] has been applied to model the breaking of the axisymmetric properties first in Reversed Field Pinches [2-4] and more recently in tokamaks [5-8]. In stellarator experiments, very long-lasting oscillations with low toroidal mode number $n$ have been previously reported [9-11]. Previous theoretical and simulation research related to this subject include nonlinear resistive MHD computations of the LHD device that yield saturated solutions [12], the generation of current-driven magnetic island structures when the rotational transform exceeds $1 / 2$ in the $\mathrm{CTH}$ (Compact Toroidal Hybrid) torsatron-tokamak hybrid with the interesting feature that initial $m / n=10 / 5$ structures coalesce to develop a lower order $m / n=2 / 1$ chain [13] and fixed boundary internal nonlinearly MHD stable 2-field period quasiaxisymmetric stellarator configurations have been calculated with the NSTAB code [14]. The deformations in that work retain the underlying 2-fold periodicity of the device proposed. In this Letter, 3D free boundary ideal magnetohydrodynamic (MHD) equilibrium states are presented for the first time with the VMEC code [1] that develop visible edge distortions that break the periodicity imposed by the coil system in a nominally 4-field period Helias reactor device. We contend that the deformations observed constitute nonlinear saturated ideal magnetohydrodynamic (MHD) interchange structures with low toroidal mode number $n$ driven by the interaction of the pressure gradient with the magnetic field line curvature. The Helias Advanced Stellarator concept investigated displays quasi-isodynamic properties $[17,18]$ which are essentially unaltered by the periodicity-breaking distortions at reactor-relevant values of $\langle\beta\rangle$.

The measurement of very long-lasting low order toroidal mode number oscillations in stellarators [9-11] and many other toroidal devices [19-23] provides the motivation to theoretically model this activity and therefore develop a physics understanding of the phenomena observed. In particular, coherent modes that spontaneously break the toroidal periodicity have been observed in the TJ-II stellarator [9]. Computation of 3D MHD equilibrium states with the VMEC code imposes nested magnetic flux surfaces [1]. The states that are computed represent ideal magnetohydrodynamics because the model precludes magnetic field lines breaking and reconnecting. The Helias configuration represents one of the most attractive options for nuclear power production based on the stellarator concept, hence we concentrate on a nominally 4-field system of this type to investigate the formation of energetically favourable solutions of the MHD equilibrium problem that break the 4 -fold periodicity imposed by the confining coil set.

The plasma energy $\mu_{0} W=\iiint d^{3} x\left[B^{2} / 2+\mu_{0} p(s) /(\Gamma-\right.$ 1 )] (where $\boldsymbol{B}$ is the magnetic field, $p(s)$ is the plasma pressure and is a function of the radial variable $s$ $(0 \leq s \leq 1)$ which is proportional to the enclosed toroidal magnetic flux function $\Phi, \Gamma$ is the adiabatic index) is minimised with respect to an artificial time variable employing a steepest descent energy minimisation scheme. A Green's function technique is applied to evolve the plasma-vacuum interface to obtain free boundary MHD equilibria where the vacuum magnetic fields are calculated using Biot-Savart's Law from all toroidal and poloidal field coils discretised into finite sized filaments. In free boundary mode, initial guesses for the Fourier amplitudes of $R$ (the distance from the major axis) and $Z$ (the height above the midplane) at the magnetic axis and at the last closed magnetic flux surface (LCFS) must be provided. Though the coil set dictates 4-fold periodicity around the torus, we relax this constraint to include all toroidal mode numbers in the range $-20 \leq n \leq 20$ and poloidal mode numbers $0 \leq m \leq 11$. Typically, the 4 -fold periodic Fourier components of $R \equiv \sum_{m, n} R_{m, n} \cos (m \theta-n \phi)$ and $Z \equiv \sum_{m, n} Z_{m, n} \sin (m \theta-n \phi)$ (stellarator symmetry is assumed) at the plasma-vacuum interface are chosen 
as $R_{0,0}=17.7, R_{0,4}=0.72, R_{1,0}=2.15, R_{1,4}=0.99$, $Z_{0,0}=0, Z_{0,4}=0.33, R_{1,0}=2.448, Z_{1,4}=1.33$ (dimensions in $\mathrm{m}$ ). Initial edge periodicity-breaking components are prescribed though the parameter $\delta_{i}$, such that $R_{1,1}=Z_{1,1}=\delta_{i}$ and $R_{1,-1}=Z_{1,-1}=-\delta_{i}$.

All calculations performed in this article have vanishing toroidal current within each flux surface $2 \pi J(s)=0$ and pressure profile prescribed as $p(s)=p(0)(1-s)(1-$ $\left.s^{4}\right)$. The rotational transform profiles $\iota(s)$ for the Helias reactor configuration examined at $\langle\beta\rangle=0.5 \%$ and $\langle\beta\rangle=5 \%$ are plotted as a function of $s$ in Fig. 1. Note that $\iota=1$ is outside the plasma; the main low order resonances that break the 4 -fold periodicity of the coils are $n / m=10 / 11,9 / 10,7 / 8,6 / 7$ and $5 / 6$.

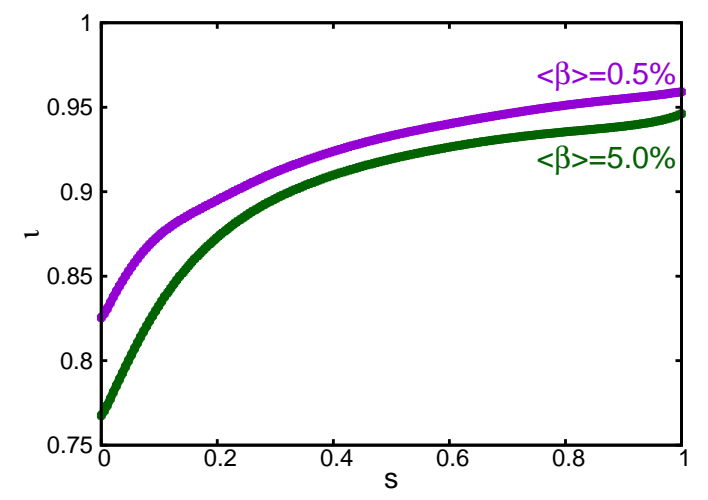

FIG. 1. The rotational transform profiles in a nominally 4field period Helias reactor configuration at $\langle\beta\rangle=0.5 \%$ and $\langle\beta\rangle=5 \%$ in which the periodicity imposed by the coils is not enforced.

The shape of the LCFS for the Helias reactor system under consideration at each of the tear-drop cross sections throughout one toroidal transit are projected onto one plane in Fig. 2 at $\langle\beta\rangle=0.5 \%, 2 \%$ and $4 \%$. There are visible deformations of the LCFS in this range of $\langle\beta\rangle$ values. The Plasma-Vacuum Interface shape at the bean, tear-drop and triangular cross sections over 1 toroidal transit at $\langle\beta\rangle=5.0 \%$ shows the small corrugation of the edge surface at each superimposed plane in Fig. 3.

To address the question for the motivation to impose initial periodicity-breaking deformations, we perform an ideal MHD stability investigation of Helias reactor equilibria constrained to satisfy the 4-fold periodicity imposed by the external coils with respect to the $n=1$ family of modes with the TERPSICHORE code $[15,16]$. Convergence studies of the configuration as a function of the inverse number of radial grid points squared is plotted at $\langle\beta\rangle=0.6 \%, 1.6 \%$ and $2.6 \%$ in Fig. 4. Marginal stability at infinitesimal mesh occurs for $\langle\beta\rangle=0.6 \%$ (nevertheless the system is very weakly unstable at finite mesh size). The parameter $\delta_{i}$ that we apply to generate equilibrium states without the periodicity constraint imposed consequently constitutes a proxy for the $m / n=1 / \pm 1$ components of the linear displacement vector of the lin-
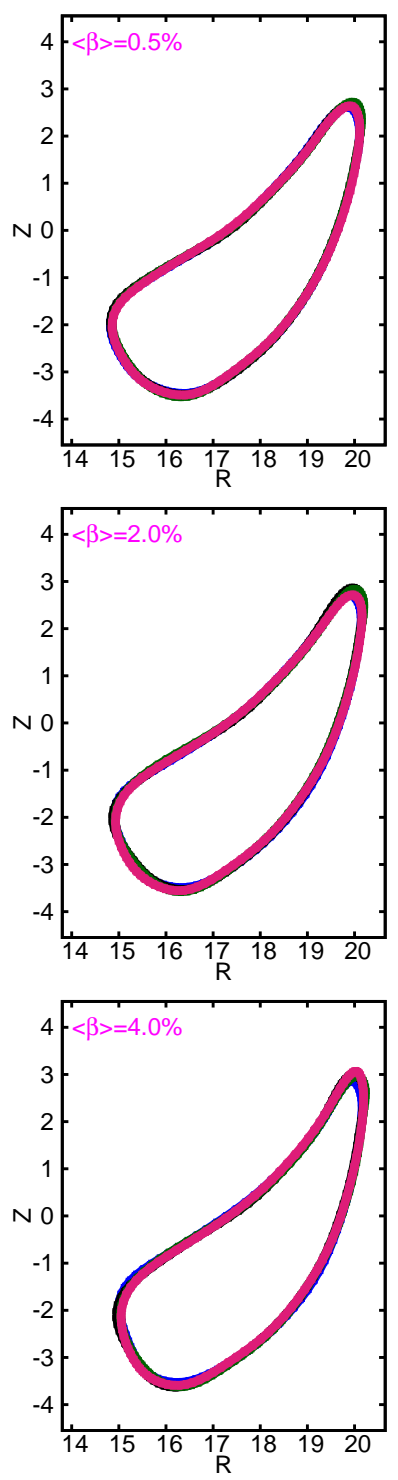

FIG. 2. The superimposed shape of the LCFS at the 4 teardrop cross sections at $\langle\beta\rangle=0.5 \%$ (top), at $2.0 \%$ (middle) and at $4.0 \%$ (bottom) in a Helias reactor system. The superimposed shapes match exactly when the 4 -fold coil periodicity constraint is imposed.

ear stability calculations at the plasma boundary. We would like to repeat that $\delta_{i}$ represents only an initial periodicity-breaking displacement; it is not a persistently applied external field like a resonant magnetic perturbation. It should be noted that error fields and eddy currents in the surrounding metallic walls will also produce periodicity-breaking perturbations that could be particularly relevant below the linear ideal MHD stability limits. The results presented in Fig. 4 justify increasing $\delta_{i}$ with $\langle\beta\rangle$. Fig. 5 shows the variation of the volume average total energy $\left\langle\left\langle p+B^{2} /\left(2 \mu_{0}\right)\right\rangle\right\rangle$ within the plasma as a function of the parameter $\delta_{i}$ that shows that the absolute minimum energy state is achieved for $\delta_{i}=0.31 \mathrm{~m}$. For 

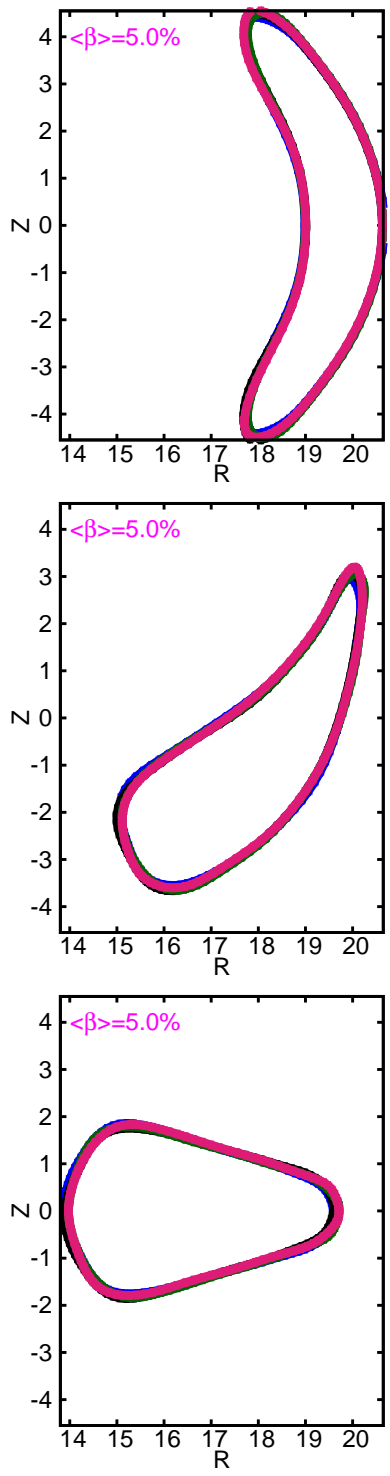

FIG. 3. The superimposed shapes of the LCFS at $\langle\beta\rangle=5.0 \%$ at the bean-shaped cross section (top), at the tear-drop cross section (middle) and at the triangular cross section (bottom) in a Helias reactor configuration. The 4 superimposed shapes match exactly at each of the cross sections when the 4 -fold coil periodicity constraint is imposed.

$\delta_{i}>0.35 \mathrm{~m}$, we have failed to obtain a converged equilibrium state with VMEC and conjecture that under such circumstances the initial state we prescribe is too far removed to achieve an equilibrium solution. For lower $\langle\beta\rangle$, the energetically favoured MHD equilibrium solutions are achieved with progressively lower values of the initial distortion $\delta_{i}$ prescribed at the plasma boundary. We define $\langle\langle\mathcal{A}\rangle\rangle \equiv \int d^{3} x \mathcal{A} / \int d^{3} x$. There is of course no guarantee that each equilibrium point in Fig. 5 actually corresponds to a stable state. However, a $2^{\text {nd }}$ variation of the volume averaged total plasma energy with respect to $\delta_{i}$ will identify the minima in Fig. 5 to represent stable states

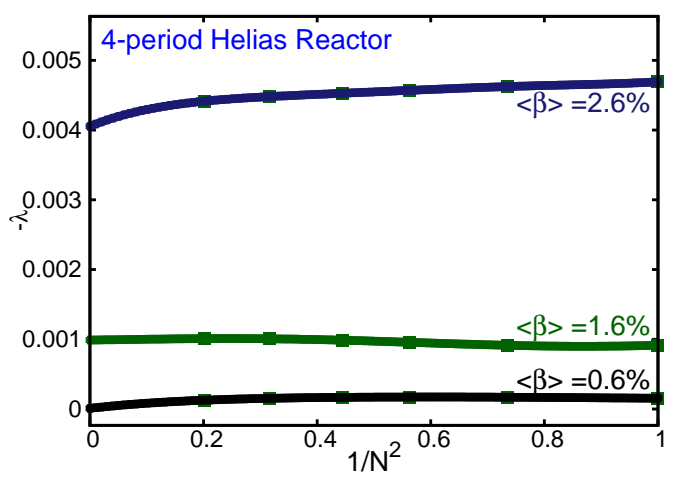

FIG. 4. The linear ideal MHD eigenvalue $\lambda$ plotted with respect to $1 / N^{2}$, where $N$ corresponds to the number of radial intervals in the plasma at different values of $\langle\beta\rangle$. A straight line identifies the convergence properties as quadratic. The 4 -fold periodicity of the underlying equilibrium states is preserved.

with respect to $m / n=1 / \pm 1$ initial deformations of the plasma boundary. The absolute minimum corresponds to the nonlinearly saturated equilibrium state at $\langle\beta\rangle=5 \%$. Hence, without actually performing an explicit stability calculation, we can infer nonlinear ideal MHD stability properties from equilibrium computations. One intriguing speculation prompted by a referee's comment is that the local minima observed at the lower values of $\delta_{i}$ in Fig. 5 constitute manifestations of the $2^{\text {nd }}, 3^{\text {rd }}$ and so forth less unstable ideal MHD states of the 4-field period constrained configuration. This merits further investigations in the future.

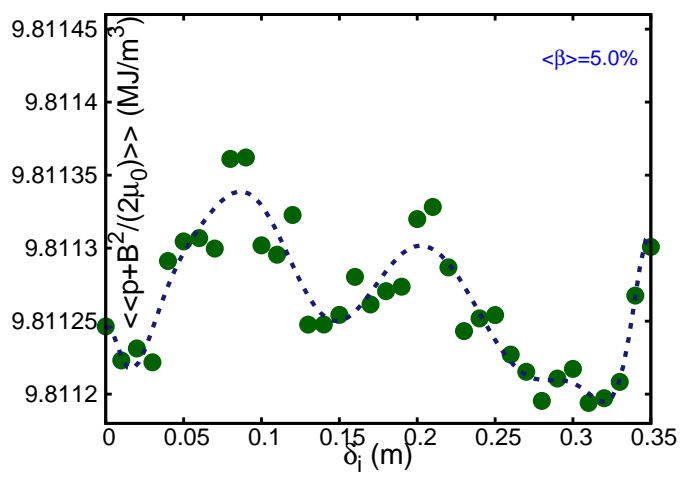

FIG. 5. The value of the volume averaged total plasma energy $\left\langle\left\langle p+B^{2} /\left(2 \mu_{0}\right)\right\rangle\right\rangle$ at the minimum energy state as a function of the initial guess of the distortion $\delta_{i}$ in a Helias reactor system at $\langle\beta\rangle=5.0 \%$. The initial Fourier amplitudes of $R$ and $Z$ corresponding to the $m=1, n= \pm 1$ components are $R_{1,1}=Z_{1,1}=-R_{1,-1}=-Z_{1,-1}=\delta_{i}$. The dotted curve represents a $15^{\text {th }}$ order polynomial fit to the simulation data points.

The minimum energy state in the range $\langle\beta\rangle=0.5 \%-$ $-5.6 \%$ is plotted in Fig. 6 . The initial guesses for the 
$m=1, n= \pm 1$ components of $R$ and $Z$ specified by $\delta_{i}$ that yield the minimum energy states at each $\langle\beta\rangle$ are identified in the plot. $\Delta\left\langle\left\langle p+B^{2} /\left(2 \mu_{0}\right)\right\rangle\right\rangle$ corresponds to the difference between $\left\langle\left\langle p+B^{2} /\left(2 \mu_{0}\right)\right\rangle\right\rangle$ evaluated at finite $\delta_{i}$ and $\delta_{i}=0$. Note that the ordinate values in Fig. 6 are negative indicating that the volume averaged total plasma energy obtained at the finite values of $\delta_{i}$ is lower than that for $\delta_{i}=0$ in the range of $\langle\beta\rangle$ explored.

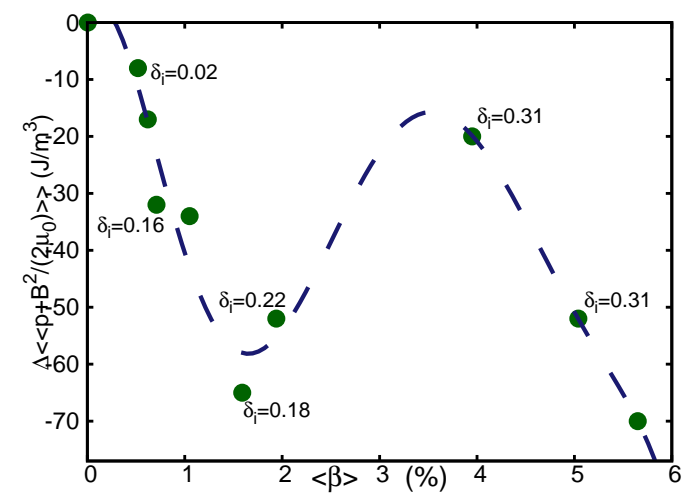

FIG. 6. The value of the difference of the volume averaged total plasma energy $\left\langle\left\langle p+B^{2} /\left(2 \mu_{0}\right)\right\rangle\right\rangle$ at finite $\delta_{i}$ (which corresponds to the minimum energy state) with that at $\delta_{i}=0$ as a function of $\langle\beta\rangle$. The values of $\delta_{i}$ are labelled at selected values of $\langle\beta\rangle$. The dashed curve represents a $6^{\text {th }}$ order polynomial fit to the simulation data points.

A Fourier decomposition of $R$ at the outermost magnetic flux surface is undertaken and displayed in Fig. 7 at $\langle\beta\rangle=0.5 \%$ and $5.0 \%$. We would like to highlight that the main resonances identified in the description of Fig. 1 that break the periodicity of the system provide only weak distortions compared with the mainly nonresonant contributions of $R_{m, n}$ that appear in Fig. 7. The spectrum is slightly broader and the amplitudes smaller in absolute magnitude at the lower $\langle\beta\rangle$ values. Though the amplitudes of the $R_{1, \pm 1}$ term increase in magnitude with $\langle\beta\rangle$, the resulting interference pattern appears not to be constructive as the corrugations of the shape of the last surface as shown in Figs. 2, 3 do not change much.

In current-free stellarators, the interaction of the pressure gradient with the magnetic field line curvature constitutes the principal driving mechanism for MHD instabilities. The structure of $2 \sqrt{g} p^{\prime}(s) \kappa \cdot \nabla s$ on a flux surface close to the edge of the plasma reveals that the most destabilising contribution (most negative) concentrates near the outside edge of one of the bean-shaped cross sections indicating an important $n=1$ distortion. Furthermore, the structure when the 4-fold periodicity is relaxed is more closely magnetic field aligned compared with the 4-field period constrained configuration where it is cross-field aligned. The comparison between the 2 cases at $\langle\beta\rangle=5 \%$ is presented in Fig. 8. Another important observation is that the range of values for $2 \sqrt{g} p^{\prime}(s) \boldsymbol{\kappa} \cdot \nabla s$ is neutral (with respect to positive and negative values) when the periodicity constraint is re-
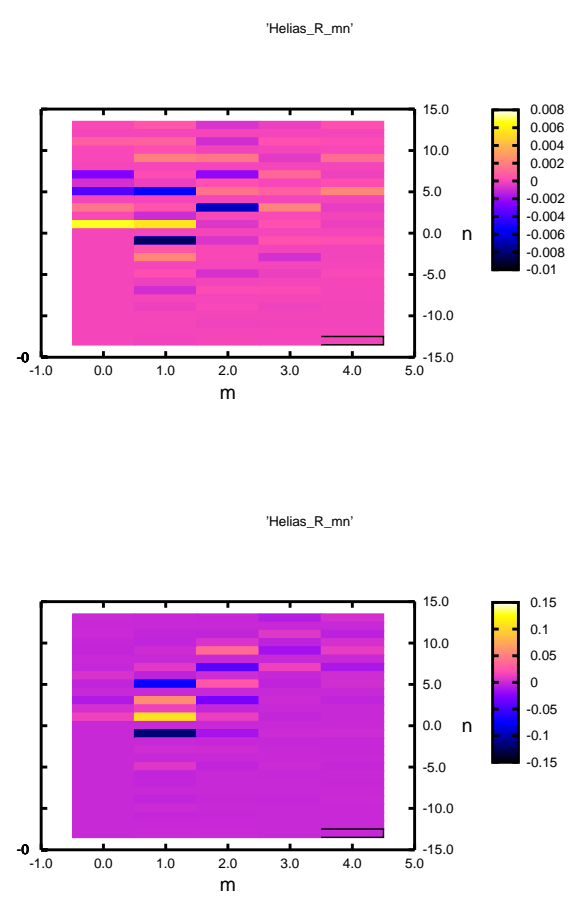

FIG. 7. The dominant Fourier components of $R$ on the LCFS in a Helias reactor system at the minimum energy state at $\langle\beta\rangle=0.5 \%$ (top) and $\langle\beta\rangle=5.0 \%$ (bottom) poloidal mode number $m$ and toroidal mode number $n$ phase space. To visualize the periodicity-breaking Fourier terms, the dominant 4 -fold periodic components of $R$ are suppressed. The main periodicity-breaking terms have significant amplitude for relatively low $m, n$ values. Consequently, we limit the display to the range $0 \leq m \leq 4$ and $-13 \leq n \leq 13$. The Fourier amplitudes $R_{m, n}$ are normalized to the $R_{1,0}$ term.

laxed indicating marginal stability. However, it tends to be more negative when the 4-fold periodicity is enforced suggesting that this configuration is linearly unstable to ideal MHD. The periodicity-breaking deformations constitute a saturated ideal MHD instability state.
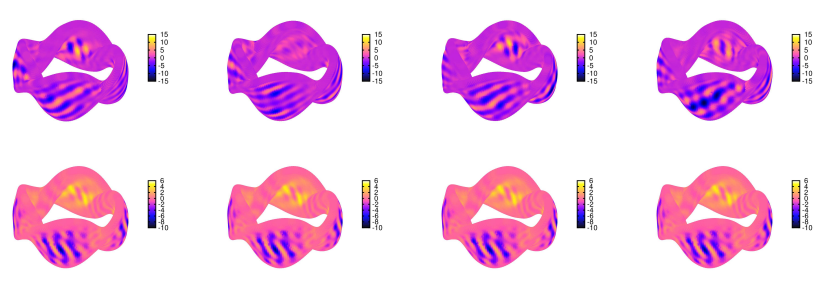

FIG. 8. The distribution of the interaction of the pressure gradient with the magnetic field line curvature $2 \sqrt{g} p^{\prime}(s) \kappa \cdot \nabla s$ on a magnetic flux surface close the edge of the plasma at $\langle\beta\rangle=5 \%$. The top row represents the equilibrium state with unconstrained periodicity. The bottom row represents the equilibrium state constrained to satisfy the 4-fold periodicity of the coils.

The Helias reactor configuration examined approaches 
conditions of quasi-isodynamicity $[17,18]$. The poloidal closure of the contours of the $2^{\text {nd }}$ adiabatic invariant $\mathcal{J}$ in a polar plot for particles with pitch angles that vary from deeply to barely trapped represents a good measure of the confinement of fast ions and of neoclassical transport properties in general. Essentially particles that reside in the phase space volume of poloidally closed $\mathcal{J}$ contours do not drift far from their birth flux surfaces and as a result should remain well confined in the $3 \mathrm{D}$ Helias $B$ field $[17,18]$. We have found that the relaxation of the periodicity exacted by the external coils does not modify in a significant way the contours of the $\mathcal{J}$ for different particle pitch angles. A comparison of the $\mathcal{J}$ behavior for the general equilibrium case and the 4 -fold periodic case at $\langle\beta\rangle=5 \%$ demonstrates that both are very similar, as shown in Fig. 9. Thus energetic particle confinement and neoclassical transport should not be seriously impacted whether periodicity is enforced or not, however this requires verification in future work.
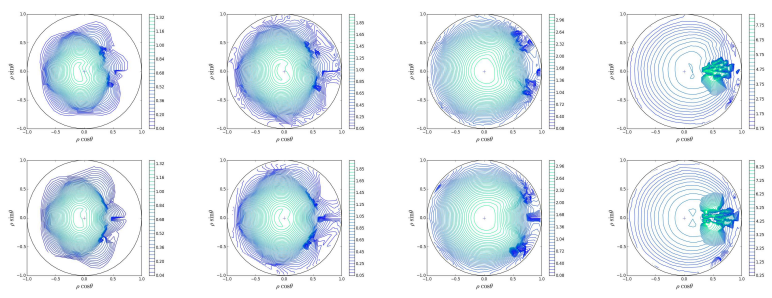

FIG. 9. The contours of the second adiabatic invariant $\mathcal{J}$ in polar coordinate representation for a Helias reactor configuration at $\langle\beta\rangle=5 \%$. The radial variable is $\rho=\sqrt{s}$ and $\theta$ is the poloidal angle. The columns correspond to reference magnetic field values $B_{\text {ref }}=4.55 T, 4.6 T, 4.7 T$ and $4.8 T$ from left to right, respectively. The particle pitch angle correspond to $\lambda \equiv \mu / \mathcal{E}=1 / B_{\text {ref }}$. The top row represents the equilibrium state with unconstrained periodicity. The bottom row represents the equilibrium state constrained to satisfy the 4 -fold periodicity of the coils.

We report, for the first time, free boundary stellarator equilibria using the VMEC code with novel 3D distortions to the plasma-vacuum interface that break the underlying periodicity imposed by the external magnetic confinement coils of a nominally 4-field period Helias reactor. The edge corrugations occur in the range $0.5 \%<\langle\beta\rangle<5.6 \%$ investigated. The Fourier spectrum of $R$ and $Z$ at the LCFS is dominantly $m=1$, $n= \pm 1$ at $\langle\beta\rangle=5 \%$. The $m / n=1 / \pm 1$ structures are nonresonant as $\iota_{\max }<0.95$. We contend that these geometric deformations correspond to saturated ideal MHD interchanges driven by the interaction of the pressure gradient with the magnetic field curvature. The $2 \sqrt{g} p^{\prime}(s) \boldsymbol{\kappa} \cdot \nabla s$ structure confirms a $n=1$ modulation around the torus. The quasi-isodynamic properties of the Helias configuration we have investigated are not significantly altered by the periodicity-breaking corrugations we report, thus we surmise that fast particle confinement and neoclassical transport will not be affected. The edge distortions are very benign (even at high $\langle\beta\rangle>5 \%$ ) compared with other confinement concepts. These conditions augur very favorably for the Helias advanced stellarator system as a power producing fusion energy reactor concept.

The simulation model we have applied constrains the magnetic surfaces to remain nested. Our work thus opens new avenues of research like the consideration of more general equilibrium approaches that allow for the formation of periodicity-breaking magnetic islands and internal stochastic regions [24-26] (typically these solvers use a VMEC solution as an initial guess). Also, nonlinear kinetic theory can be invoked to examine the generation of finite frequency oscillations using the VMEC equilibria we have computed.

The work was supported in part by the Swiss National Science Foundation and by the Spanish Government (project ENE2014-52174-P) within the framework of the EUROfusion Consortium and received funding from the Euratom research and training programme 20142018 under grant agreement No 633053. The views and opinions expressed herein do not necessarily reflect those of the European Commission. We thank Dr. S. P. Hirshman for providing us with the VMEC code, Dr. M. Drevlak for using routines of the ROSE code, Dr. H. F. G. Wobig (RIP) and Dr. J. Kisslinger for the Helias coils. We also acnkowledge the anonymous referees for insightful suggestions.

*wilfred.cooper@epfl.ch

[1] S.P. Hirshman et al., Comput. Phys. Commun. 43, 143 (1986).

[2] R. Lorenzini et al., Nature Physics 5, 570574 (2009)

[3] D. Terranova et al., Plasma Phys. Contr. Fusion 52, 124023 (2010).

[4] J.D. Hanson et al., Nucl. Fusion 53, 083016 (2013).

[5] W.A. Cooper et al., Phys. Rev. Lett. 105035003 (2010).

[6] W.A. Cooper et al., Nucl. Fusion 53, 073021 (2013).

[7] W.A. Cooper et al., Phys. Plasmas 23040701 (2016).

[8] A. Wingen et al., Nucl. Fusion 56, 016013 (2016).

[9] M.A. Ochando et al., in Proceedings of the $42^{\text {nd }}$ EPS Conference in Plasma Physics, Lisbon, Portugal, 2015.

[10] Y. Takemura et al., Nucl. Fusion 52, 102001 (2012).

[11] T. Bando, S. Ohdachi, Y. Suzuki, R. Sakamoto, Y. Narushima, Y. Takemura, K.Y. Watanabe, S. Sakakibara, X.D. Du, G. Motojima, K. Tanaka, T. Morisaki, and LHD Experiment Group, Phys. Plasmas 25, 012507 (2018).

[12] K. Ichiguchi et al., Nucl. Fusion 43, 1101 (2003).

[13] M. Schlutt, C. C. Hegna, C. R. Sovinec, S. F. Knowlton and J. D. Herbert, Nucl. Fusion 52, 103023 (2012).

[14] P. Garabedian, Proc. Natl. Acad. Sci. USA 103, 1923219236 (2006)

[15] D.V. Anderson, W.A. Cooper, R. Gruber, S. Merazzi and U. Schwenn, Int. J. Supercomp. Appl. 4, 34 (1990).

[16] W.A. Cooper, Plasma Phys. Control. Fusion 34, 1011 (1992).

[17] J. Nührenberg, Plasma Phys. Control. Fusion 52, 124003 
(2010)

[18] H. Mynick, Phys. Plasmas 13, 058102 (2006).

19] J.H. Harris et al., Phys. Rev. Lett. 53, 2242 (1984).

[20] J. Manickam et al., Nucl. Fusion 27, 1461 (1987).

21] M.P. Gryaznevich et al., Nucl. Fusion 48, 084003 (2008).

[22] L.F. Delgado-Aparicio et al., Nucl. Fusion 53, 043019 (2013).

[23] R-B. Zhang et al., Plasma Phys. Control. Fusion 56, 095007 (2014).
[24] Y. Suzuki, N. Nakajima, K. Watanabe, Y. Nakamura and T. Hayashi, Nucl. Fusion 46, L19 (2006).

[25] H. Peraza-Rodriguez, J.M. Reynolds-Barredo, R. Sanchez, J. Geiger, V. Tribaldos, S.P. Hirshman and M. Cianciosa, Phys. Plasmas 24, 082516 (2017).

[26] J. Loizu, S.R. Hudson and C. Nührenberg, Phys. Plasmas 23, 112505 (2016). 